\section{Opening keynote}

\section{$\mathrm{KL}$ - Keynote lecture}

\section{KL01 ENDING THE HIV/AIDS PANDEMIC: FROM SCIENTIFIC ADVANCES TO PUBLIC HEALTH IMPLEMENTATION}

doi:10.1136/sextrans-2013-051184.0001

A S Fauci. National Institute of Allergy and Infectious Diseases, NIH, Bethesda, MD, United States

Extraordinary advances in basic and clinical HIV/AIDS research over three decades have led to the development of effective interventions, particularly for treating and preventing HIV infection. These interventions have clearly been shown to be efficacious in clinical trials and effective when properly implemented "on the ground." For example, combination antiretroviral therapy (ART) dramatically improves the health and longevity of HIV-infected people. Adult male circumcision is highly effective in protecting heterosexual men from acquiring HIV infection. Preventing motherto-child transmission of HIV infection with antiretroviral drugs has been highly successful. Antiretroviral drugs also play a critical role in preventing the sexual transmission of HIV; thus, treatment of HIV-infected people decreases dramatically the likelihood that they will transmit the virus to others. In addition, pre-exposure prophylaxis with antiretroviral drugs delivered orally or at the genital mucosa reduces the risk of acquiring HIV infection. Significant scientific challenges remain in HIV research, notably in developing a cure and a vaccine for HIV infection; however, the scientifically proven interventions currently available offer unprecedented opportunities to make major gains in the fight against HIV/AIDS. In many settings, the implementation of these interventions in a cohort or well-defined population has already resulted in impressive results, strongly indicating that global implementation scale-up could have the effect of dramatically changing the trajectory of the HIV/AIDS pandemic and ultimately leading to the end of the AIDS pandemic. We are on scientifically solid ground that this goal is achievable with a major global commitment. 